\title{
Vorläufiges Abkürzungsverzeichnis
}

\author{
A. C., AppCas \\ AChILLES-Greiff-(Bearb $)^{21}$ \\ AcP \\ AHKG \\ Am. J. Comp. I. \\ Amtsvorm. \\ Ann. Inst. Dr. Int. \\ Anziloter, Corso \\ AöR \\ Ariz. L. Rev. \\ Arminjon, Précis ${ }^{3}$ \\ AWD \\ BadRpr \\ v. BAR, IPR \\ v. Bar, Theorie und Praxis ${ }^{2}$ \\ Barazetti, IPR \\ Bartin, Principes \\ Batiffol, Traité \\ BayObLGZ
}

BB

Beitzke, Jur Personen

Bek

BerGesVR

Bergmann-Ferid

Betrieb

BGBl

BGE

BGHZ

BlIntPr.

\section{$\mathbf{A}$}

Appeal Cases (Law Reports)

AChilles-GreifF, Bürgerliches Gesetzbuch 21. Aufl. Berlin 1958

Archiv für die civilistische Praxis (1.1818-149. 1944; 150. $1948 / 49 \mathrm{ff}$.)

Gesetz der Allierten Hohen Kommission

The American Journal of Comparative Law. (1. 1952ff.) Der Amtsvormund, Rundbrief d. Dt. Instituts f. Vormundschaftswesen (24. 1951/52ff.)

Annuaire de l'Institut de Droit International. (1. 1877ff.) Anzilotit, Corso di lezioni di diritto internazionale (Dirittoprivato) Roma 1918

Archiv des öffentlichen Rechts (1. 1866ff.)

The Arizona Law Review

Arminjon, Précis de droit international privé, 3. Aufl. Paris 1947

Außenwirtschaftsdienst des Betriebs-Beraters (4. 1958ff.)

\section{B}

Badische Rechtspraxis und Annalen der Großh. Badischen Gerichte (1. 1899-102. 1936)

v. BAR, Lehrbuch des Internationalen Privat- und Strafrechts, Stuttgart 1892

v. Bar, Theorie und Praxis des Internationalen Privat. rechts, 2 Bde. 2. Aufl. Hannover 1889

Barazertr, Das internationale Privatrecht im Bürgerlichon Gesetzbuch für das Deutsche Reich, Hannover 1897 Bartin, Principes de droit international privé selon la loi et la jurisprudence francaises, 3 Bde. Paris 1930/32/35 Batiffor, Droit international privé, 4. Aufl. Paris 1967 (Sammlung von) Entscheidungen des Bayer. Obersten Landesgerichts in Zivilsachen (1. 1901-34. 1934/35; N. F. 1. $1950 \mathrm{ff}$.)

Der Betriebs-Berater (1. 1946ff.)

Beitzke, Juristische Personen im International-Privatrecht und Fremdenrecht, München, Berlin 1938.

Bekanntmachung

Berichte der Deutschen Gesellschaft für Völkerrecht (1. $1957 \mathrm{ff}$.)

Brrgmann-Ferid, Internationales Ehe- und Kindschaftsrecht 3. Aufl. Frankfurt a. M. (Loseblattausgabe)

Der Betrieb (1. $1948 \mathrm{ff}$.)

Bundesgesetzblatt (1950 ff)

Entscheidungen des Schweizerischen Bundesgerichtes aus dem Jahre ... (1. 1875ff.)

Entscheidungen des Bundesgerichtshotes in Zivilsachen (1. $1951 \mathrm{ff}$. )

Blätter für intermationales Privatrecht (= Beilage zur Leipziger Zeitschrift) (1. 1926-6. 1931) 
Bull. dr. tchéeosl.

BVerfGE

BW

$\mathrm{CC}$

Cheshire, PrIntLaw?

Clunet

Codigo Bustamante

Colum. L. Rev.

D.

DA

DGVZ

DiCEY.Morris, Conflict ${ }^{8}$

Diss

DJZ

DNotZ

DöLLE, IPR

D. $P$.

DR

Droit Int. Famille

DRpflege

DRspr.

DRZ

DÜRINGER-HACHENBURG

DVO

Eggar

Ehrenzweia, Conflict

ENNECCERUS-NIPPERDEY ${ }^{15}$

ErbbauVO

Erman-Bearb.)

FamGB

FamRÄG

FebozzI, DirIntPriv ${ }^{2}$

Ferid-Firsching

FGB
Abkürzungs-Verzeichnis

Bulletin de droit tchécoslavoque (6. 1948ff.)

Entscheidungen des Bundesverfassungsgerichts (1. 1952ff.)

Burgerlijk Wetboek (Niederlande)

\section{C}

Code civile, codigo civil, codice c vile

Cheshire, Private international law, 7. Aufl. London 1965

Journal du droit international (1. 1874ff.)

Bustamante y Sirven, Projet de code de droit international Privé, Paris 1925

Columbia Law Review (1. $1901 \mathrm{ff}$.)

\section{D}

Recueil Dalloz de doctrine, de jurisprudence et de législation $(1945-1954 ; 1957-1964)$

Dienstanweisung

Deutsche Gerichtsvollzieher-Zeitung (1. 1881-64. 1944; 65. $1950 \mathrm{ff}$.)

Dice Y-MoRris, The conflict of laws, 8. Aufl. London 1967

Dissertation

Deutsche Juristenzeitung (1. 1896-41. 1936)

Deutsche Notar-Zeitschrift (1. 1901-44. 1944; 1950ff.)

DöLLE, Internationales Privatrecht, Karlsruhe 1968

Dalloz. Recueil périodique et critique de jurisprudence de législation et de doctrine $(1825-1940)$

Deutsches Recht (1. 1933-15. 1945)

Le droit international privé de la famille en France et en Allemagne, Tübingen, Paris 1955

Deutsche Rechtspflege (1. 1936-4. 1939)

Deutsche Rechtsprechung (1948ff.)

Deutsche Rechts-Zeitschrift (1. 1946-5. 1950)

Düringer-Hachenborg, Das Handelsgesetzbuch vom 10. Mai 1897 (unter Ausschluß des Seerechts) auf der Grundlage des Bürgerlichen Gesetzbuches 3. Aufl. Mannheim 1930 -

Durchführungsverordnung

\section{$\mathbf{E}$}

Egate, Familienrecht in: Kommentar zum Schweizerischen ZGB, Zürich 2. Aufl. $1936 \mathrm{ff}$.

Ehrenzweig, A treatise on the conflict of laws, St. Paul 1962

Enneccerus-NipPerdey, Allgemeiner Teil des Bürgerlichen Rechts 15. Aufl. Tübingen 1959/60

VO ü. d. Erbbaurecht v. 15. 1.1919 (RGBl. S. 72)

Erman, Handkommentar zum Bürgerlichen Gesetzbuch 4. Aufl. Münster 1967

\section{I"}

Familiengesetzbuch der DDR v. 20. 12. 1965 (GBl. DDR 1966 I, 1) Gesetz zur Vereinheitlichung und Änderung familienrechtlicher Vorschriften (Familienrechtsänderungsgesetz) v. 11. 8. 1961 (BGB1. I S. 1221)

Fedozzi, Il diritto internazionale privato 2. Aufl. Padova 1939

Ferid-Firschina, Internationales Erbrecht, München 1955 - (Loseblattausgabe)

siehe FamGB 
Ficker, Grundfragen

Foro It.

frz

Fschr

Fischer-Henle-Titze 14

Frankenstein, Code

FRANKENSTEIN, IPR

GaMILISChEG, IntArbR

GBl

GBl. DDR

GebharD

Giur. Comp. D. I. P.

GLU

Graulich, Principes

GOODRICH-SCOLES, Handbook ${ }^{4}$

griech

Gruchot

Grzybowski, Soviet PrIntLaw

GUTZWILLER, IPR

HABICHT

HansGZ

HansRGZ

HansRZ

HAuslG

HessRspr

HRR

JbIntRVerk

JBl. Rh-Pf

JbOstR

J.-Cl. Dr. Int.

I. C. L. Q.

J. C. P.
Abkürzungs-Vorzeichnis

Ficker, Grundfragen des deutschen interlokalen Rechts, Berlin, Tübingen 1952

Il Foro Italiano (1. $1876 \mathrm{ff}$.)

französisch

Festschrift

Fischer-Henle-Titze, Bürgerliches Gesetzbuch 14. Aufl. München 1932

Frankenstein, Projet d'un code européen de droit inter. national privé. Leiden 1950

Frankenstein, Internationales Privatrecht (Grenzrecht) 4 Bde. Berlin 1926 -

\section{G}

GamillscheG, Internationales Arbeitsrecht, Berlin 1959 Gesetzblatt

Gesetzblatt der Deutschen Demokratischen Republik (1949ff.)

Die Gebhardschen Materialien veröffentlicht von NIEMEYER, Zur Vorgeschichte des Internationalen Privatrechts, München, Leipzig 1915

Giurisprudenza Comparate di Diritto Internazionale Privato (1. 1937-12. 1956)

Sammlung von civilrechtlichen Entscheidungen des k. k. Obersten Gerichtshofes (1. 1859-34. 1901; N. F. $1.1900-$ 18. 1919

Graulich, Principes de droit international privé, Paris 1961

GOODRTCH-Scoles, Handbook of the conflict of laws 4. Auf. St. Paul 1964

griechisch

Beiträge zur Erläuterung des Deutschen Rechts (1. 185773. 1933

Grzybowski, Soviet Private International Law, Leiden 1965

GUTZwILleR, Internationalprivatrecht, Berlin 1931

$\mathbf{H}$

Habicht, Internationales Privatrecht nach dem Ein. führungsgesetze zum bürgerlichen Gesetzbuche, Berlin 1907 Hanseatische Gerichtszeitung (1. 1880-48. 1927)

Hanseatische Rechts- und Gerichtszeitschrift (11. 1928 26. 1943)

Hanseatische Rechtszeitschrift für Handel, Schiffahrt ... (1. 1918-10. 1927)

Gesetz über die Rechtsstellung heimatloser Ausländer im Bundesgebiet vom 25. 4. 1951 (BGBl. I S. 269)

Hessische Rechtssprechung (1. 1900/01-35. 1935)

Höchstrichterliche Rechtsprechung (4. 1928-19. 1942)

J

Jahrbuch für den Internationalen Rechtsverkehr (1912/13) Justizblatt Rheinland-Pfalz (1. $1947 \mathrm{ff}$.)

Jahrbuch für Ostrecht (1. 1960 ff.)

Juris-classeur de droit international, Paris 1952 - (Loseblattausgabe)

The International and Comparative Law Quarterly (1. $1952 \mathrm{ff}$.)

Juris Classeur périodique (1. $1927 \mathrm{ff}$.) 


JFG
JFGErg
JherJb
JIR
Ill. L. Rev.
IntRDipl
IPRspr

JR
ital
JurJb
JW
JZ
IzRspr

KAHN, Abhandlungen

KEGEL, IPR 2

KEIDEL, FGG'

KGJ

KLANG, $_{\text {(Bearb) }}{ }^{2}$

KöнLER, IPR ${ }^{3}$

KrönIG, IP-Zust in dFG

LAINÉ, Introduction

Law \& Contemp. Prob.

Leflar, Conflict

LemaIRE, IPR

Lent-Habscheid, FGG4

Lerebours-PigeonNIÈre/ Loussouar, DIP

LEvis, Dt. int. Entmündigungsrecht

LEWAID, IPR

LEwald, Règles générales LM

LUNz, IPR
Abkürzungs-Verzeichnis

Jahrbuch für Entscheidungen in Angelegenheiten der freiwilligen Gerichtsbarkeit (1. 1924-23. 1943)

Entscheidungen des Kammergerichts (und des Oberlandesgerichts München) in Kosten-, Straf-, Miet- und Pachtschutzsachen) (1. 1924-23. 1944)

Jherings Jahrbücher der Dogmatik des bürgerlichen Rechts (1. 1857-90. 1942)

Jahrbuch für internationales (und ausländisches öffentliches) Recht (1. 1948ff.)

Illinois Law Review (1. 1906/07-46. 1951/52)

Internationales Recht und Diplomatie (1. 1956ff.)

Die deutgche Rechtsprechung auf dem Gebiete des internationalen Privatrechts im Jahre (in den Jahren ...) (1926/27-1934, 1945/1949ff.)

Juristische Rundschau (1. 1925-11. 1935; 1. 1947ff.)

italienisch

Juristen-Jahrbuch (1. 1960 ff.)

Juristische Wochenschrift (1. 1872-68. 1939)

Juristenzeitung (6. $1951 \mathrm{ff}$.)

Sammlung der deutschen Entscheidungen zum interzonalen Privatrecht

\section{$\mathbf{K}$}

KAHN, Abhandlungen zum internationalen Privatrecht 2 Bde. München und Leipzig 1928

Kegel, Internationales Privatrecht, 2. Aufl. München und Berlin 1964

KeIDEL, Freiwillige Gerichtsbarkeit 9. Aufl. München, Berlin 1967

Jahrbuch für Entscheidungen des Kammergerichts in Sachen der freiwilligen Gerichtsbarkeit, in Kosten-, Stempel- und Strafsachen (1. 1881-53. 1922)

Kommentar zum allgemeinen Bürgerlichen Gesetzbuch 2. Aufl. Wien $1950 \mathrm{ff}$.

KömLER, Internationales Privatrecht 3. Auf. Wien-New York 1966

KæöNıG, Die international-privatrechtliche Zustündigkeit in der freiwilligen Gerichtsbarkeit, Hamburg 1936

L

LAINÉ, Introduction au droit international privé ... 2 Bde. Paris 1888

Law and Contemporary Problems (1. 1933/34ff.)

LEFLAR, The law of conflict of laws, Indianapolis 1959

Lemaire, Nederlands Internationaal Privatrecht, Leiden 1968

Lent-Habscheid, Freiwillige Gerichtsbarkeit, 4. Aufl. München-Berlin 1962

Lerfbours-Pigeonntere/Loussouarn, Droit intermational privé, 8. Aufl. Paris 1962

LEvis, Das internationale Entmündigungsrecht des Deutschen Reiches, Leipzig 1906

Lewadd, Das deutsche internationale Privatrecht, Leipzig 1931

Lewald, Règles générales des conflits de lois, Basel 1941 Nachschlagewerk des Bundesgerichtshofes (1951 ff.)

Lonz, Internationales Privatrecht, 2 Bde. Berlin-0 $1961 / 64$ 
Abkürzungs-Verzeichnis

Makarov, Quellen²

Marty-Raynaud, Droit Civil

MatThine, IntZust

MaUnz-DÜRIG-HeRzoG ${ }^{3}$

MDR

MecklZ

Melchior, Grundlagen

M. L. R.

MRG

Moser, Vertragsabschluß

NAG

ndl

NdsRpfl

Neunaus, Grundbegriffe

Neumann, IPR

Neumeyer, Gemeinrechtliche, Entwicklung

NEUMEYER, IPR ${ }^{2}$

Niboyet, Traité

Niedner ${ }^{2}$

Nieme yer, IPR des BGB

Nifme yer, Vorgeschichte NIEMEYERSZ

N. J.

NJW

Nussbaum, Grundzüge

Nussbaum, IPR

N. Y. L. F.
M

Makarov, Quellen des Internationalen Privatrechts, 2 Bde. Berlin/Tübingen 1953/1960

Marty-Raynaud, Droit Civil, Paris 1956 -

Matтhies, Die deutsche internationale Zuständigkeit, Frankfurt 1955

Maunz-Dürig-Herzog, Grundgesetz, Kommentar. 3. Aufl. München 1969

Monatsschrift für Deutsches Recht (1. 1947ff.)

Mecklenburgische Zeitschrift für Rechtspflege und Rechtswissenschaft (1. 1881-55. 1939)

Melchior, Die Grundlagen des deutschen internationalen Privatrechts, Berlin, Leipzig 1932

The Modern Law Review (1. 1937/38ff.)

Militärregierungsgesetz

Moser, Vertragsabschluß, Vertragsgültigkeit und Parteiwille im internationalen Obligationenrecht, St. Gallen 1948

$\mathbf{N}$

(Schweizerisches) Bundesgesetz betreffend die zivilrechtlichen Verhältnisse der Niedergelassenen und Aufenthalter v. 25. 6. 1891

niederländisch

Niedersächsische Rechtspflege (1. $1947 \mathrm{ff}$.)

Neuhaus, Die Grundbegriffe des Internationalen Privatrechts, Berlin/Tübingen 1962

Nedmane, Internationales Privatrecht in Form eines Gesetzentwurfs Nach Motiven und Materialien Berlin 1896 Neumeyer, Die gemeinrechtliche Entwicklung des internationalen Privat- und Strafrechts bis Bartolus 2 Bde. München 1901/1916

NedMe Yer, Internationales Privatrecht 2. Aufl. München 1930

Nıво yет, Traité de droit international privé francais 6 Bde. 1. und 2. Auflage. Paris $1944 \mathrm{ff}$.

Nicder, Das Einführungsgesetz zum BGB, Kommentar, 2. Aufl. Berlin 1901

Niemeyer, Das internationale Privatrecht des BGB, Berlin 1901

siehe GEBHARD

Zeitschrift für internationales Privat- und Strafrecht (1. 1891-52. 1938)

Nederlandse Jurisprudentie (1913ff.)

Neue Juristische Wochenschrift (1. 1947/48ff.)

Nussbaum, Grundzüge des internationalen Privatrechts, München 1952

Nussbaum, Deutsches Internationales Privatrecht, Tübingen 1932

New York Law Forum (1. 1955ff.)

o

(österreichisches) Allgemeines bürgerliches Gesetzbuch vom 1.6. 1811

Österreichische Notariatszeitung (81. $1949 \mathrm{ff}$.

Osteuropa-Recht (1. $1955 \mathrm{ff}$.)

Entscheidungen des Obersten Gerichtshofs für die Britische Zone in Zivilsachen (1. 1949-4. 1950) 


\section{Abkürzungs-Verzeichnis}

OLGRspr

Pagenstecher, Entscheidungseinklang

Palandt (-Bearb) ${ }^{28}$

Pas.

Pillet, Principes

PlaANCK $^{3}$

poln

portugies

Poullet, Manuel ${ }^{3}$

PrALR

Priese-Rebentrost, WG

PStG

\author{
RAAPE, IPR $^{5}$ \\ RABel, Conflict 2

\section{RabelsZ} \\ Rec. des Cours \\ Recht \\ Réczer, IPR \\ ReithmanN, Vertragsrecht \\ Rép. Dalloz Dr. Int. \\ Rép. Lapradelle-Niboyet \\ Restatement 2nd
}

Rev. crit.

RGBl

RGWarn

RGZ

RIEZIER, IZPR

RIGAUX, DIP

Riv.Dir.Int.

Riv.Not.

Row

Rpfleger

RvglHWB

RzW
Die Rechtssprechung des Oberlandesgerichts auf dem Gebiete des Zivilrechts hrsg. v. Mugdan und Falkmann (1. 1900-46. 1928)

P

Pagenstecher, Der Grundsatz des Entscheidungseinklangs im internationalen Privatrecht Mainz 1951

Palandt, Bürgerliches Gesetzbuch, 29. Aufl. München 1969

Pasicrisie belge (1864ff.)

Pillet, Principes de droit international privé, Paris

Planck, Kommentar zum BGB, 3. Aufl. Berlin 1905

polnisch

portugiesisch

Poullet, Manuel de droit international privé belge, 3. Aufl. 2 Bde. Bruxelles 1947

Allgemeines Landrecht für die preußischen Staaten, gültig ab 1. 6. 1794

Priese-Rebentrost, Kommentar zum Wechselgesetz, Iserlohn 1949

Personenstandsgesetz v. 3. 11. 1937 (RGBl. I S. 1146) i. d. F. v. 8. 8. 1957 (BGBl. I S. 1125)

\section{$\mathbf{R}$}

RAAPE, Internationales Privatrecht, 5. Aufl, Berlin und Frankfurt 1961

RABEL, Conflicts of law: a comparative study, 4 Bde., 2. Aufl. Berlin 1958 -

(Rabels) Zeitschrift für ausländisches und internationales Privatrecht (1. $1927 \mathrm{ff}$.)

Recueil des Cours (1. $1923 \mathrm{ff}$.)

Das Recht (1. 1897-48. 1944)

RÉczeI, Internationales Privatrecht, Budapest 1960

ReithmanN, Internationales Vertragsrecht, Köln 1963

Répertoire de droit international, 2 Bde. Paris 1968/69

Répertoire de droit international, Paris 10 Bde. 1929-1931

Restatement of the law (Conflict of Laws), second edition. As adopted and promulgated by the American Law Institute at Washington, D. G.; St. Paul, Minn. $1957 \mathrm{ff}$.

Revue critique de droit international privé (36. $1947 \mathrm{ff}$.)

Reichsgesetzblatt

Rechtsprechung des Reichsgerichts soweit sie nicht in der amtlichen Sammlung der Entscheidungen des RG abgedruckt ist, hrsg. v. Warneer (1. 1908-33. 1941)

Entscheidungen des Reichsgerichts in Zivilsachen (1. 1880. 172. 1945)

RiezLer, Internationales Zivilprozeßrecht und prozessuales Fremdenrecht, Berlin Tübingen 1949

RIGaUx, Droit international privé, Bruxelles 1968

Rivista di Diritto Internationale (1. 1906 - 35. 1943; $36.1953 \mathrm{ff}$

Rivista del Notariato

Recht in Ost und West (1. 1957ff.)

Der deutsche Rechtspfleger (56./57. 1948/1949 ff)

Rechtsvergleichendes Handwörterbuch für das Zivil- und Handelsrecht des In- und Auslandes hrsg. v. Schlegel. Berger. 7 Bde. Berlin 1929-1940

Rechtssprechung zum Wiedergutmachungsrecht (1. 1949/ $50 \mathrm{ff}$.) 
Abkürzungs-Verzeichnis

$\mathbf{S}$

s.

$\mathrm{SaBl}$

SächsArch

SAVATIER, DIP ${ }^{2}$

SAvigny, System VIII

SchG

SchlHA

SCrNitzer, Hdb I ${ }^{4}$

Schw.Jb.Int.R.

SchwOR

SchwZGB

SeuffArch

SeuffBl

SJZ

SOFRGEL-(Bearb) $)^{9}$

span

STANZ, WG ${ }^{14}$

Staudinger-(Bearb) ${ }^{11}$

StAZ

Stein-Jonas-Poht.e, ZPO $^{19}$

SteindoRfF, Sachnormen

Story, Commentaries ${ }^{8}$

Stumberg, Principles ${ }^{3}$

SwoBoda

Szászy, PrIntLaw

Thomas-Putzo, $\mathrm{ZPO}^{3}$

türk

U.Chi.L.Rev

U.Pa.L.Rev.

VerschÄndG

VerschG
Recueil Sirey (1791-1954; 1957-1964)

Sammelblatt für Rechtsvorschriften des Bundes und der Länder (1. 1949/50 ff.)

Sächsisches Archiv für Bürgerliches Recht und Prozeß (1. 1891-15. 1905)

Savatier, Cours de droit international privé, 2. Aufl. Paris 1953

Saviany, System des heutigen Römischen Rechts Bd. 8 Berlin 1849

Scheckgesetz v. 14. 8. 1933 (RGBl. I S. 597)

Schleswig-Holsteinische Anzeigen (194. $1947 \mathrm{ff}$.)

Schntтzer, Handbuch des internationalen Privatrechts 4. Aufl. Bd I Basel 1957

Schweizerisches Jahrbuch für Internationales Recht (1. 1944ff.)

(Schweizerisches) Obligationenrecht v. 30. 3. 1911/18. 12. 1936

Das Schweizerische Zivilgesetzbuch v. 10.12.1907

SeUfFerts Archiv für Entscheidungen der obersten Gerichte in den deutschen Staaten (1. 1847-98. 1944)

SEUFFERTs Blätter für Rechtsanwendung (1. 1836-78. 1913)

Schweizerische Juristen-Zeitung (1. 1904ff.)

SoERgel-Siebert, Bürgerliches Gesetzbuch. Mit Einführungsgesetz und Nebengesetzen. 9. Auf. Stuttgart 1959-1963 10. Aufl. $1967 \mathrm{ff}$. spanisch

spanisch

Stanz, Wechselgesetz, Kommentar, 14. Aufl. Berlin 1952 Staddinger, Kommentar zum Bürgerlichen Gesetzbuch. Mit Einführungsgesetz, und Nebengesetzen, 11. Aufl. Berlin $1957 \mathrm{ff}$.

Zeitschrift für Standesamtswesen (1. 1921-24. 1944 N.F. 1. 1948; dann: Das Standesamt)

Stein-Jonas-Pohle, Kommentar zur Zivilprozeßordnung, 19. Auf. Tübingen $1964 \mathrm{ff}$.

Steindorf, Sachnormen im internationalen Privatrecht. Frankfurt a.M. 1958

Story, Commentaries on the conflict of laws, ... 8. Aufl. Boston 1883

Stumbera, Principles of conflicts of Laws, 3. Aufl. Brooklyn 1963

swoBoda, Das internationale Recht der freiwilligen Gerichtsbarkeit Diss. München 1934

SzÁszy, Private international law in the European people's democracies. Budapest 1964

\section{$\mathbf{T}$}

Thomas-Putzo, Zivilprozeßordnung 3. Auf. München 1968 türkisch

$\mathbf{U}$

The University of Chicago Law Review (1. 1933/34ff.)

University of Pennsylvania Law Review (1.1852 ff.)

$\mathbf{v}$

Gesetz zur Änderung von Vorschrift end. Verschollenheitsrecht v. 15. 1. 1951 (BGBl. I S. 59)

Verschollenheitsgesetz i.d.F. v. 15. 1. 1951 (BGBl. I. S. 63) 


\author{
VerschKonv. \\ VIscher, Vertragsrecht \\ VOBl \\ VOBl.Br.Z
}

\section{WALKER, IPR ${ }^{5}$ \\ $\mathrm{WbVR}^{1}$}

\section{$\mathrm{WbVR}^{2}$}

\section{WG}

WM

WO

WOLFF, IPR ${ }^{3}$

Wolfe, PrIntLaw ${ }^{2}$

WürttZ

Yale L.J.

ZaöRV

ZblFG

ZblJR

ZGB

ZHR

Ziv Cod

ZITELMANN, IPR

ZLR

ZöLLER, ZPO ${ }^{10}$

ZRpfBay

ZVglRWiss

ZZP

\section{Abkürzungs-Verzeichnis}

Konvention der Vereinigten Nationen über die Todeserklärung Verschollener v. 6. 4. 1950 (BGBl. II 1955 S. 706) Vischer, Internationales Vertragsrecht Bern 1962 Verordnungsblatt

Verordnungsblatt für die Britische Zone (1947-1949)

\section{$\mathbf{W}$}

WaJker, Internationales Privatrecht 5. Aufl. Wien 1934 Wörterbuch des Völkerrechts und der Diplomatie. Hrsg. v. Strupp 3 Bde. Berlin und Leipzig $1924 \mathrm{~b}-1929$

Wörterbuch des Völkerrechts 2. Aufl. (hrsg. v. SchLochAUER) 3 Bde, Berlin 1960-1962

Wechselgesetz v. 21. 6. 1933 (RGBl. I S. 399)

Wertpapier-Mitteilungen. Teil IV (1. 1947ff.)

Wechselordnung i.d.F. v. 3. 6. 1908 (RGBl S. 326)

Worff, Das Internationale Privatrecht Deutschlands 3. Aufl. Berlin, Göttingen, Heidelberg 1954

WoLfF, Private International Law 2. Aufl. Oxford 1950 Württembergische Zeitschrift für Rechtspflege und Verwaltung (ab 25. 1932: für Verwaltung und Verwaltungspflege (1907-31. 1938)

$\mathbf{Y}$

The Yale Law Journal (1. 1891/92ff.)

\section{$\mathbf{Z}$}

Zeitschrift für ausländisches öffentliches Recht und Völkerrecht (1. 1929-12. 1944; 13. 1950/51 ff.)

Zentralblatt für freiwillige Gerichtsbarkeit und Notariat (1. 1900/01-22. 1921/22)

Zentralblatt für Jugendrecht und Jugendwohlfahrt (1. 1908-36. 1944; 37. 1950ff.)

Zivilgesetzbuch

Zeitschrift für das gesamte Handelsrecht und Wirtschaftsrecht (1. 1858-110.1944; 111. 1946/48ff.)

Polnischer Zivilkodex v. 23. April 1964

Zitelmani, Internationales Privatrecht 2 Bde. Leipzig $1897 / 1912$

Zeitschrift für das gesamte Luftrecht (1. 1926-2. 1928/29)

ZöLLER, Zivilprozeßordnung 10. Aufl. München 1968

Zeitschrift für Rechtspflege in Bayern (1. 1905-30. 1934)

Zeitschrift für vergleichende Rechtwissenschaft (1. 1878 bis 55.1942 ; $56.1953 \mathrm{ff}$.)

Zeitschrift für Zivilprozeß (1. 1879-63. 1943;64. 1950/ $51 \mathrm{ff}$. 